Given the need to improve the efficien$c y$ of data transfer, there are requirements to ensure their reliability and quality under interference. One way to improve data transfer efficiency is to use noise-resistant codes, which include a closed-form expression of the Fibonacci code, a parity check code, and a constant weight code. The result of applying these types of coding produces interference-resistant end-to-end processing and transmission of information, which is a promising approach to improving the efficiency of telecommunications systems in today's environment. This paper reports the estimation of the error detecting code capability of the Fibonacci code in a closed-form expression, as well as its comparative characteristic with a parity check code and a constant weight code for a binary symmetrical channel without memory. To assess an error detecting capability of the Fibonacci code in a closed-form expression, the probability of Fibonacci code combinations moving to the proper, allowable, and prohibited classes has been determined. The comparative characteristic of the indivisible error-detecting codes is based on an average probability method, for the criterion of an undetectable error probability, employing the MATLAB and Python software. The method has demonstrated the simplicity, versatility, and reliability of estimation, which is close to reality. The probability of an undetectable error in the Fibonacci code in a closed-form expression is $V=5 \times 10^{-7}$; in a code with parity check, $V=7.7 \times 10^{-15}$; and in a constant weight code, $V=1.9 \times 10^{-15}$, at $p_{10}=3 \times 10^{-9}$. The use of the average probability method makes it possible to effectively use indivisible codes for detecting errors in telecommunications systems

Keywords: average probability method, indivisible code, error-detecting code, undetectable error, reliability
UDC 004.312.43.052

DOI: $10.15587 / 1729-4061.2020 .218076$

\section{ESTIMATING THE INDIVISIBLE ERROR DETECTING CODES BASED ON AN AVERAGE PROBABILITY METHOD}

\author{
O. Boryse n ko \\ Doctor of Technical Sciences, Professor* \\ E-mail: O.Borysenko@ekt.sumdu.edu.ua \\ S. Mat se nko \\ $\mathrm{PhD}$, Leading Researcher* \\ Communication Technologies Research Center** \\ E-mail: S.Matsenko@ekt.sumdu.edu.ua \\ A. Novhorodtsev \\ $\mathrm{PhD}$, Associate Professor* \\ E-mail: A.Novhorodtsev@ekt.sumdu.edu.ua \\ O. Ko b y a k o v \\ $\mathrm{PhD}$, Associate Professor* \\ E-mail: O.Kobyakov@ekt.sumdu.edu.ua \\ S. Spolitis \\ $\mathrm{PhD}$, Professor \\ Institute of Telecommunications \\ Communication Technologies Research Center** \\ E-mail: Sandis.Spolitis@rtu.Iv \\ V. Bobrovs \\ $\mathrm{PhD}$, Professor \\ Institute of Telecommunications** \\ Azenes str., 12, Riga, Latvia, LV-1048 \\ E-mail: Vjaceslavs.Bobrovs@rtu.Iv \\ *Department of Electronics and Computer Technics \\ Sumy State University \\ Rymskoho-Korsakova str., 2, Sumy, Ukraine, 40007 \\ **Riga Technical University \\ Azenes str., 12, Riga, Latvia, LV-1048
}

\section{Introduction}

The modern telecommunication systems must meet the requirements for the reliable transfer, high-speed performance, processing, and storage of information. One of the main ways to improve the noise immunity of telecommunication systems is to use error control codes. At the same time, telecommunication systems may employ a variety of anti-interference codes, starting from the simplest ones such as a parity-checking code to more sophisticated cyclical codes that can correct multiple errors. Various error control codes, such as cyclic block codes, which include, for example, Bose-Chaudhuri-Hocquenghem codes, Reed-Solomon codes [1-3], as well as the codes with a low density of parity checks [3, 4], can be applied to solve such tasks. Also wellknown today are, for example, polar codes [5] and turbocodes [6] with artificial redundancy.

The use of redundant information contained in the encoded words is the main criterion for any interference-resistant codes, which is typically introduced into them artificially. There is also another type of redundant coding that uses natural redundancy in the coded words. Employing natural redundancy codes requires far less hardware to synthesize encoding and decoding devices while using simpler algorithms to accomplish these tasks.

In addition, the application of codes with natural redundancy in telecommunication systems, such as optical data transmission systems, can be greatly enhanced by using 
the codes with artificial redundancy, employing the hybrid types of error control codes. In this way, one can achieve more effective indicators to protect transmitted and processed data from errors, while using relatively less complex algorithms in encoding and decoding devices for hardware implementation. At the same time, codes with natural redundancy are capable of executing end-to-end control of data. The end-to-end data control can be enabled both in digital devices and in transmission systems, which greatly improves the efficiency of telecommunication systems. The application of codes involving artificial redundancy does not allow for a through data control; it is designed only for error control tasks, either in communication channels or in information processing systems. This creates the task to effectively evaluate codes with natural redundancy, such as the indivisible error detection codes, for their further application in telecommunication systems or digital devices.

\section{Literature review and problem statement}

Indivisible error-detecting codes with natural redundancy include, for example, the codes generated by such number systems as factorial [7, 8], binomial [9, 10], and Fibonaccian [11].

The factorial number system makes it possible to generate code permutations that can be used for interference-resistant coding, with natural redundancy. The downside of factorial codes, which, like permutations, are multi-digit codes, is that there is not a high level of effective interference-resistant coding, even with additional artificial coding [7]. Paper [8] examines the factorial codes involving the Euclid and Hamming metrics. The authors report a comparative analysis of the probability of an undetectable error and the residual probability of mistaken factorial codes. The study results show that the factorial codes using the Hamming metric are more effective. However, there remain unresolved issues related to the comparative analysis of factorial codes with other types of coding and the methods for evaluating the error-detectable capability of indivisible codes.

The binomial number system makes it possible to generate a binomial code and ensures high interference resistance while making it possible to detect binary errors of type $0 \rightarrow 1$ and $1 \rightarrow 0$. Binomial codes can be used effectively for end-to-end control, processing, and transmission of information. This type of coding is a rather complex structure; its interference resistance is recommended to be used in solving functional problems, for example, to generate compositions, to generate and sort combinatorial configurations [9]. Work [10] evaluated the noise immunity of binomial modified codes for the criterion of the probability of an undetectable error for symmetrical and asymmetric channels with independent errors. It is shown that at a certain level of interference in the communication channel, it is more appropriate to use binomial modified codes, which provide greater reliability of the transmitted data compared to the application of linear cyclic block codes. The results show that the use of a binomial modified code ensures a high error-detectable capability in communication channels with high levels of asymmetry. However, there remain unresolved issues related to the comparative estimation of binomial modified codes with the Fibonacci code in a closed-form expression, with a parity-check code, and a constant weight code.

Constant weight codes are also error-detecting codes. The advantage of a code with a permanent weight is the quick and efficient finding of errors while processing information. This code consists of $k$ 1's and $n$ - $k 0$ 's where $n$ is the length of the code combination. Identifying the error is the discrepancy between the number of $n-k 0$ 's and the number of 1's $k$ in the code combination [12]. Study [13] assessed the probability of an undetectable error in binary $(n, 26, m)$ non-linear codes with a permanent weight using a binary symmetrical channel. However, there remain unresolved issues related to the comparative estimation of indivisible error-detecting codes with other types of coding.

Among the well-known anti-interference codes that detect errors, the Fibonacci code can be also used effectively in practice. Its peculiarity is that the code consists of numbers generated by the Fibonaccian numbering system [14-16]. Fibonacci codes are the indivisible noise-resistant codes, with a natural redundancy in their structure, and are able to detect asymmetric errors not only in data channels but also in synthesized digital devices that operate on their basis. For example, such as fast-acting Fibonacci counters, fractal deciphers, ciphers. Paper [17] examines the informational and arithmetic frameworks for the construction of Fibonacci's $p$-codes and the "golden" $p$-proportion codes, as well as the synthesis of digital devices based on them. Work [17] considers the meta-Fibonacci number system based on the meta-Fibonacci sequences; it is a generalization of the Zeckendorf number system.

Study [19] reports the estimate of an error detecting capability of the Fibonacci code in a closed-form expression but this estimate is generalized and does not use probabilistic code characteristics or comparative estimates with other types of coding. Typically, transitions are used from one form of a Fibonacci code representation, minimal, to another, maximal, and back, requiring the operations of sweep and convolution. This complicates the process of decoding the Fibonacci numbers and increases its time, so the Fibonacci code in a closed-form expression was selected to this end.

The Fibonacci code in a closed-form expression is close to a standard binary code and, therefore, is the easiest one, in terms of structure, among the codes derived from noise-resistant number systems. The Fibonacci code is capable of detecting errors in both processing and transfer of information, which means that it can execute end-to-end control in telecommunication systems $[19,20]$. However, these errors' type is $0 \rightarrow 1$. Therefore, the Fibonacci code in a closed-form expression is most effective in asymmetric channels for processing and transmitting information, which is quite common in practice. In addition, if necessary, this asymmetry can be easily compensated for by the introduction of the simplest interference-resistant codes with artificial reservations, thereby obtaining a cumulative effect of improved interference resistance.

Paper [21] reports a method for evaluating indivisible error-detecting codes. It shows that a given estimate can be applied to any indivisible codes. However, there remain unresolved issues related to the estimation of the above error detecting codes, which were not previously more thoroughly researched using an average probability method. Thus, it is difficult to use different types of coding in telecommunication systems and compare them with other types of coding. The option of overcoming the relevant difficulties may be to use the average probability method to evaluate codes, which makes it possible to objectively investigate and compare codes, thereby rendering relevance to appropriate research. The above suggests that it is expedient to undertake a study 
into the estimation of indivisible error-detecting codes based on an average probability method. Such an assessment is necessary and relevant in order to compare the error detecting capability of indivisible error-detecting codes and their effective practical application considering the advent of new coding methods.

\section{The aim and objectives of the study}

The aim of this study is to evaluate the indivisible error-detecting codes based on an average probability method using the examples of the Fibonacci code, a parity check code, and a permanent-weight code.

This would provide an opportunity to improve the efficiency of data processing in information and computing systems and their further transmission in telecommunication systems. In practice, the probabilities of errors in the applied indivisible codes make it possible to assess the loss and distortion of information, thereby enabling them to be compensated, for example, by reprocessing and transmitting it, or to estimate the material costs of obtaining the unreliability of information. In addition, by comparing interference resistance, one can choose a more effective indivisible code based on this parameter.

To accomplish the aim, the following tasks have been set:

- to assess the anti-interference in processing and transmitting information by the Fibonacci code in a closed-form expression based on an average probability method;

- to assess the anti-interference in processing and transmitting information by a parity check code and by an constant weight code; to perform a comparative assessment of the interference resistance of the specified indivisible codes.

This involves assessing the probability of proper transitions of code combinations $C$, the probability of undetectable erroneous transitions of code combinations $V$, the probability of erroneous transitions of code combinations that are detected $Z$.

\section{Materials and methods to study the Fibonacci code}

The Fibonacci number system makes it possible to generate a Fibonacci code in a closed-form expression; it consists of Fibonacci numbers whose weights are a sequence of the Fibonacci numbers 1, 1, 2, 3, 5, 8, .., $F_{n}$. Each sequence of numbers is determined from the following equation [14-16].

$$
F_{n}=F_{n-1}+F_{n-2} .
$$

It follows from equation (1) that each subsequent element of the Fibonacci series is equal to the sum of its two preceding elements. The quantitative value of the Fibonaccian numbers is set by a numbering function whose weights are the Fibonacci numbers [14-16]:

$$
N=a_{n} F_{n}+a_{n-1} F_{n-1}+\ldots+a_{i} F_{i}+\ldots+a_{1} F_{1}
$$

where $a_{i} \in\left\{\begin{array}{ll}0 & 1\end{array}\right\}$ is the binary digit of the $i$-th bit in the positional representation of a number; $n$ is the length of the code; $F_{i}$ is the weight of the $i$-th bit, which is equal to the $i$-th Fibonacci number. The abbreviated form of equation (1) is written in the following form given in [14-16]:

$$
N_{a}=a_{n} a_{n-1} \ldots a_{i} \ldots a_{1} .
$$

In equation (2), there is no zero low-order bit of the function. The zero bit of the low order of the combination is always 0 , with the code combination weight ratio equal to 1 . The zero low-order bit of the code combination is not recorded and does not affect the result of the summation. Thus, this representation defines the Fibonacci code in a closed-form expression. For example, the numbers 25, 54, and 33 are represented by the Fibonacci code in a closed-form expression in the form given in Table 1.

Table 1

Fibonacci code in a closed-form expression

\begin{tabular}{|c|c|c|c|c|c|c|c|c|}
\hline Bit number & 8 & 7 & 6 & 5 & 4 & 3 & 2 & 1 \\
\hline Bit weight & 21 & 13 & 8 & 5 & 3 & 3 & 2 & 1 \\
\hline$N=25$ & 0 & 1 & 0 & 0 & 0 & 1 & 0 & 1 \\
\hline$N=54$ & 1 & 0 & 1 & 0 & 1 & 0 & 1 & 0 \\
\hline$N=33$ & 0 & 1 & 0 & 1 & 0 & 1 & 0 & 1 \\
\hline
\end{tabular}

The range of Fibonaccian numbers is determined from the equation given in [14-16]:

$$
P=F_{n}+F_{n-1},
$$

where $F_{n}$ is the weight of the $n$-th bit of a Fibonacci number; $F_{n-1}$ is the weight of the $n-1$ bit of a Fibonacci number.

Using two binary numbers, the range of Fibonacci numbers equals $P_{2}=1+1=2$, for $P_{3}=2+3=5$, for $P_{4}=3+5=8$.

Fibonacci codes prohibit having two unities side by side, which is a sign of an error. If there are 3 neighboring unities in the code combination, the average 1 can be corrected by inverting it to zero, resulting in a correction of the error in the error detecting code. Thus, the Fibonacci code in a closed-form expression is able not only to detect errors but also to correct some of them.

\section{A mathematical model of the average probability} method to evaluate the indivisible error-detecting codes

The noise immunity estimation of indivisible codes for a binary symmetrical channel without memory was proposed in work [21].

Fig. 1 shows the transformation graph of the allowable code combinations into the class of proper code combinations $C$, the class of prohibited code combinations $Z$, and the class of allowable code combinations $V$.

Underlying the mathematical model is a condition that any of the $M$ allowable code combinations, transmitted along a binary symmetrical channel without memory, out of the total number of $N>M$ code combinations, can move to the following classes of code combinations, such as:

1) a class of proper code combinations $C$;

2) a class of $Z=N-M$ prohibited code combinations;

$3)$ a class of $V=M-1$ allowable code combinations with undetectable errors, where $M$ is the number of allowable code combinations; $N$ is the total number of code combinations.

The probability of a proper transition is determined from the equation given in [21]: 


$$
C=\sum_{i=1}^{M} P_{i} p_{i}^{i}
$$

where $P_{i}$ is the probability that an information source generates the $i$-th code combination; $p_{i}^{i}$ is the probability that the $i$-th code combination is properly transferred into the $i$-th code combination.

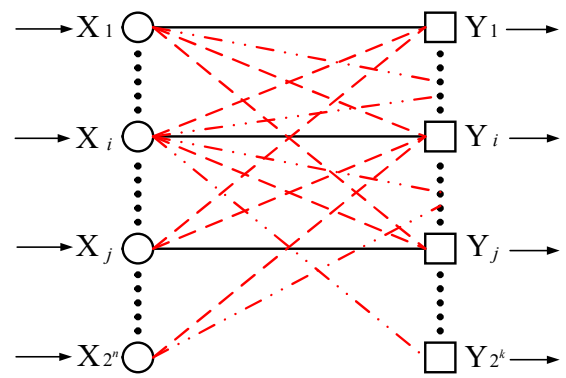

Fig. 1. Transformations of the allowable code combinations into the class of proper code combinations $C$, the class of prohibited code combinations $Z$, and the class of allowable code combinations $V$

The probability of undetectable erroneous transitions is determined from the equation given in [21]:

$$
V=\sum_{i=1}^{M} P_{i} p_{i}^{u}
$$

where $p_{i}^{u}$ is the possibility that the $i$-th code combination is erroneously transferred into a class of code combinations that are not detected.

The probability of an erroneous transition is determined from the equation given in [21]:

$$
p_{i}^{u}=\sum_{j=1, j \neq i}^{M} p_{i, j}^{u}
$$

where $p_{i, j}^{u}$ is the probability that the $i$-th code combination is erroneously transferred into the $j$-th allowable code combination.

The probability of the error detected is determined from the equation given in [21]:

$$
Z=\sum_{j=1}^{M} P_{i} p_{i}^{d}
$$

where $p_{i}^{d}$ is the possibility that the $i$-th code combination is erroneously transferred into the class of code combinations that are detected.

The probability of an erroneous transition is determined from the equation given in [21]:

$$
p_{i}^{d}=\sum_{j=M+1}^{N} p_{i, j}^{d},
$$

where $p_{i, j}^{d}$ is the possibility that the $i$-th code combination is erroneously transferred into the $j$-th prohibited combination.

The probability of code word transitions is determined from the equation given in [21]:

$$
C+V+Z=1 \text {, }
$$

where $C, V$ and $Z$ are the probabilities that code combinations are transferred into the proper, allowable, and forbidden ones.

The basic characteristic of a code is the magnitude of the probability that the allowable code combination is transferred into another allowable one (the probability of an undetectable error) [21].

$$
P_{e r r}=V=1-Z-C \text {. }
$$

Therefore, in order to assess the noise immunity of the Fibonacci code in a closed-form expression, we shall analyze the proper transmission of data, detected, and undetectable errors. The inputs for our analysis are the probability of 0 moving to $0\left(p_{00}\right)$, and 1 - to $1\left(p_{11}\right)$. The probability of an erroneous transition of 0 to 1 , and 1 to 0 , is determined from the following ratios: $p_{01}=1-p_{00}, p_{10}=1-p_{11}$.

\section{Modeling results}

6. 1. Estimating the noise immunity in processing and transfer of information by the Fibonacci code in a closedform expression

To achieve the above goal, one needs to determine the number of the allowable and prohibited code combinations in the codes under consideration; to determine the probability of erroneous transitions $0 \rightarrow 1$ and $1 \rightarrow 0$ in code combinations, as well as the probabilities of their appearance at the input to information processing devices or a communication channel.

Table 2 gives an example of the allowable code combinations $M$ in the Fibonacci code in a closed-form expression with weights $1,2,3,5,8$, for $n=5$.

Table 2

Fibonacci code in a closed-form expression of the number with weights 1, 2, 3, 5, 8

\begin{tabular}{|c|c|c|c|c|c|}
\hline \multirow{2}{*}{ No. } & \multicolumn{5}{|c|}{ Fibonacci code } \\
\cline { 2 - 6 } & 8 & 5 & 3 & 2 & 1 \\
\hline 0 & 0 & 0 & 0 & 0 & 0 \\
\hline 1 & 0 & 0 & 0 & 0 & 1 \\
\hline 2 & 0 & 0 & 0 & 1 & 0 \\
\hline 3 & 0 & 0 & 1 & 0 & 0 \\
\hline 4 & 0 & 0 & 1 & 0 & 1 \\
\hline 5 & 0 & 1 & 0 & 0 & 0 \\
\hline 6 & 0 & 1 & 0 & 0 & 1 \\
\hline 7 & 0 & 1 & 0 & 1 & 0 \\
\hline 8 & 1 & 0 & 0 & 0 & 0 \\
\hline 9 & 1 & 0 & 0 & 0 & 1 \\
\hline 10 & 1 & 0 & 0 & 1 & 0 \\
\hline 11 & 1 & 0 & 1 & 0 & 0 \\
\hline 12 & 1 & 0 & 1 & 0 & 1 \\
\hline
\end{tabular}

Table 3 gives the prohibited code combinations $F=N-M$ in the Fibonacci code in a closed-form expression with weights $1,2,3,5,8$ for $n=5$. 
Table 3 sion of data by the Fibonacci code in a closed-form expres-

Prohibited code combinations in the Fibonacci code in a closed-form expression

\begin{tabular}{|c|c|c|c|c|c|}
\hline No. & \multicolumn{5}{|c|}{ Prohibited code combinations } \\
\hline 0 & 0 & 0 & 0 & 1 & 1 \\
\hline 1 & 0 & 0 & 1 & 1 & 0 \\
\hline 2 & 0 & 0 & 1 & 1 & 1 \\
\hline 3 & 0 & 1 & 0 & 1 & 1 \\
\hline 4 & 0 & 1 & 1 & 0 & 0 \\
\hline 5 & 0 & 1 & 1 & 0 & 1 \\
\hline 6 & 0 & 1 & 1 & 1 & 0 \\
\hline 7 & 0 & 1 & 1 & 1 & 1 \\
\hline 8 & 1 & 0 & 0 & 1 & 1 \\
\hline 9 & 1 & 0 & 1 & 1 & 0 \\
\hline 10 & 1 & 0 & 1 & 1 & 1 \\
\hline 11 & 1 & 1 & 0 & 0 & 0 \\
\hline 12 & 1 & 1 & 0 & 0 & 1 \\
\hline 13 & 1 & 1 & 0 & 1 & 0 \\
\hline 14 & 1 & 1 & 0 & 1 & 1 \\
\hline 15 & 1 & 1 & 1 & 0 & 0 \\
\hline 16 & 1 & 1 & 1 & 0 & 1 \\
\hline 17 & 1 & 1 & 1 & 1 & 0 \\
\hline 18 & 1 & 1 & 1 & 1 & 1 \\
\hline & & & & & \\
\hline
\end{tabular}

Table 4 gives the number of the allowable code combinations in the Fibonacci code in a closed-form expression for $n=3, \ldots, 32$.

Table 4

The number of allowable code combinations $M$ in the Fibonacci code in a closed-form expression for $n=3, \ldots, 32$

\begin{tabular}{|c|c|c|c|c|c|}
\hline$n$ & $M$ & $n$ & $M$ & $n$ & $M$ \\
\hline 3 & 5 & 13 & 610 & 23 & $1.214 \times 10^{4}$ \\
\hline 4 & 8 & 14 & 986 & 24 & $1.964 \times 10^{5}$ \\
\hline 5 & 13 & 15 & $1.596 \times 10^{3}$ & 25 & $5.142 \times 10^{5}$ \\
\hline 6 & 21 & 16 & $2.584 \times 10^{3}$ & 26 & $8.32 \times 10^{5}$ \\
\hline 7 & 34 & 17 & $4.181 \times 10^{3}$ & 27 & $1.346 \times 10^{6}$ \\
\hline 8 & 55 & 18 & $6.765 \times 10^{3}$ & 28 & $2.178 \times 10^{6}$ \\
\hline 9 & 89 & 19 & $1.095 \times 10^{4}$ & 29 & $5.703 \times 10^{6}$ \\
\hline 10 & 143 & 20 & $1.771 \times 10^{4}$ & 30 & $9.227 \times 10^{6}$ \\
\hline 11 & 232 & 21 & $4.637 \times 10^{4}$ & 31 & $1.493 \times 10^{7}$ \\
\hline 12 & 377 & 22 & $7.503 \times 10^{4}$ & 32 & $2.416 \times 10^{7}$ \\
\hline
\end{tabular}

Table 5 gives the quantity of numbers of the prohibited code combinations $F$ in the Fibonacci code in a closed-form expression for $n=3, \ldots, 32$.

The effectiveness of using the Fibonacci code in a closedform expression is shown on the basis of the probabilities of errors taken in the range of $10^{3}-10^{9}$, which are most used in practice.

Using expression (5), we have derived a probability dependence of the proper transmission of data $C$ of the Fibonacci code in a closed-form expression. Fig. 2 shows the graph of the probability dependence of the proper transmis- sion on $\log _{10}\left(p_{10}\right)$, at $n=9$, for:

1) $p_{10}=3 \times 10^{-4}-3 \times 10^{-9}$;

2) $p_{10}=1.7 \times 10^{-4}-1.7 \times 10^{-8}$.

Table 5

The numbers of the prohibited code combinations in the Fibonacci code in a closed-form expression

\begin{tabular}{|c|c|c|c|c|c|}
\hline$n$ & $F$ & $n$ & $F$ & $n$ & $F$ \\
\hline 3 & 3 & 13 & $7.582 \times 10^{3}$ & 23 & $8.314 \times 10^{6}$ \\
\hline 4 & 8 & 14 & $1.54 \times 10^{4}$ & 24 & $1.666 \times 10^{7}$ \\
\hline 5 & 19 & 15 & $3.117 \times 10^{4}$ & 25 & $3.336 \times 10^{7}$ \\
\hline 6 & 43 & 16 & $6.295 \times 10^{4}$ & 26 & $6.679 \times 10^{7}$ \\
\hline 7 & 94 & 17 & $1.269 \times 10^{5}$ & 27 & $1.337 \times 10^{8}$ \\
\hline 8 & 201 & 18 & $2.554 \times 10^{5}$ & 28 & $2.676 \times 10^{8}$ \\
\hline 9 & 423 & 19 & $5.133 \times 10^{5}$ & 29 & $5.355 \times 10^{8}$ \\
\hline 10 & 881 & 20 & $1.031 \times 10^{6}$ & 30 & $1.072 \times 10^{9}$ \\
\hline 11 & $1.816 \times 10^{3}$ & 21 & $2.068 \times 10^{6}$ & 31 & $2.144 \times 10^{9}$ \\
\hline 12 & $3.719 \times 10^{3}$ & 22 & $4.148 \times 10^{6}$ & 32 & $4.289 \times 10^{9}$ \\
\hline
\end{tabular}

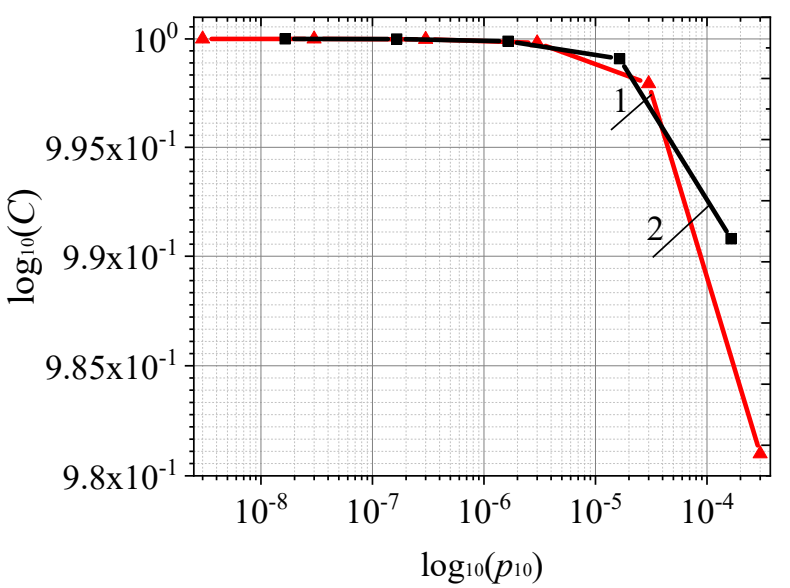

Fig. 2. Probability of the proper data transmission of the Fibonacci code in a closed-form expression: $1-p_{10}=3 \times 10^{4}-3 \times 10^{9} ; 2-p_{10}=1.7 \times 10^{4}-1.7 \times 10^{8}$

It follows from Fig. 2 that as the probability $\left(p_{10}\right)$ decreases, the probability of proper data transmission $C$ of the Fibonacci code in a closed-form expression increases. It also follows from Fig. 2 that if one uses the probabilities $p_{10}=1.7 \times 10^{4}-1.7 \times 10^{8}$, the probability of proper data transmission $C$ of the Fibonacci code in a closed-form expression is higher than when using probabilities in the range of $p_{10}=3 \times 10^{4}-3 \times 10^{9}$.

Using expression (6), we derived a probability dependence of an undetectable error $V$ of the Fibonacci code in a closedform expression on $\log _{10}\left(p_{01}\right)$. Fig. 3 shows the graph of a probability dependence of an undetectable error of the Fibonacci code in a closed-form expression on $\log _{10}\left(p_{01}\right)$, at $n=9$, for:

1) $p_{01}=3 \times 10^{3}-3 \times 10^{8}$;

2) $p_{01}=1.7 \times 10^{3}-1.7 \times 10^{7}$

It follows from Fig. 3 that as the probability value $\left(p_{01}\right)$ decreases, the probability of an undetectable error $V$ of the Fibonacci code in a closed-form expression decreases. It also follows from Fig. 3 that when one uses the probabilities $p_{01}=3 \times 10^{-3}-3 \times 10^{-8}$, the probability of an undetectable error $V$ of the Fibonacci code in a closed-form expression is greater than that when using the probabilities of data transfer in the range of $p_{01}=1.7 \times 10^{3}-1.7 \times 10^{-7}$. 


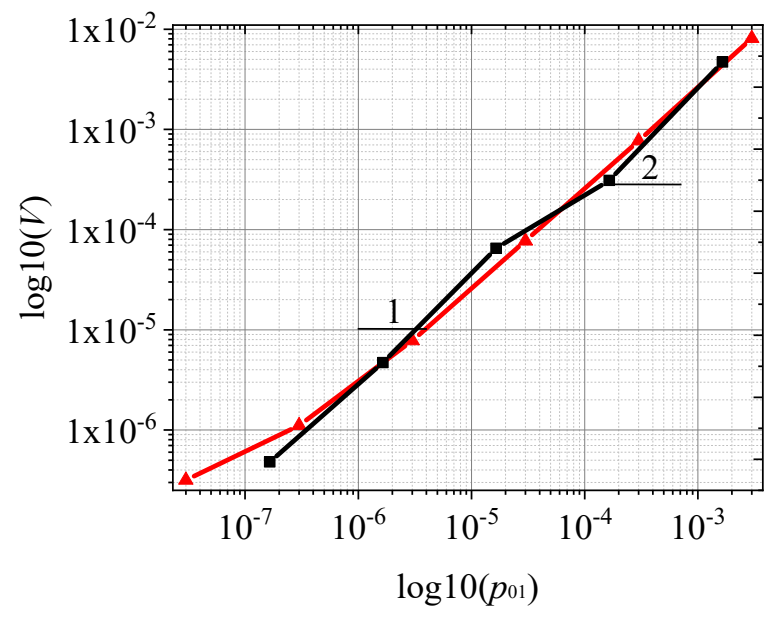

Fig. 3. Probability of an undetectable error of the Fibonacci code in a closed-form expression: $1-p_{01}=3 \times 10^{3}-3 \times 10^{8}$; $2-p_{01}=1.7 \times 10^{3}-1.7 \times 10^{7}$

Using expression (8), we derived a probability dependence of the detectable error $Z$ of the Fibonacci code in a closed-form expression on $\log _{10}\left(p_{10}\right)$. Fig. 4 shows the graph of a probability dependence of the detectable error of the Fibonacci code in a closed-form expression on $\log _{10}\left(p_{10}\right)$, at $n=9$, for:

1) $p_{10}=3 \times 10^{-4}-3 \times 10^{-9}$;

2) $p_{10}=1.7 \times 10^{-4}-1.7 \times 10^{-8}$.

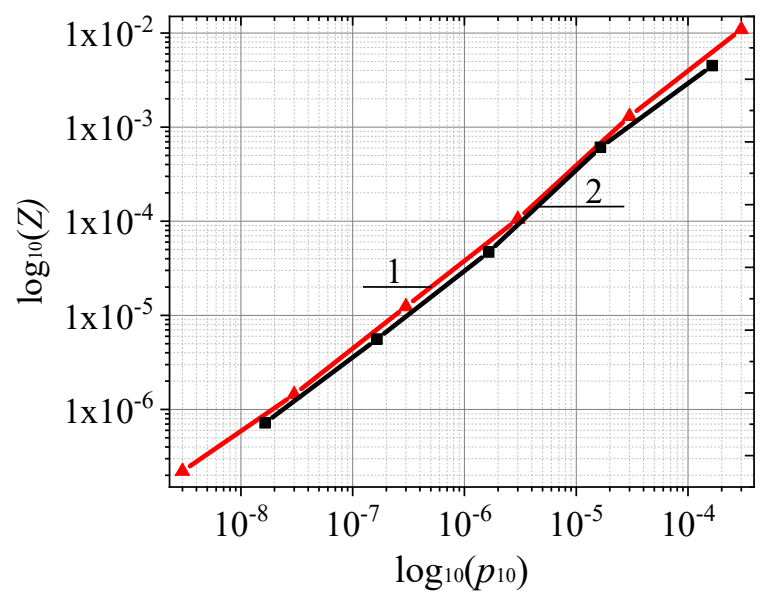

Fig. 4. Probability of a detected error of the Fibonacci code in a closed-form expression: $1-p_{10}=3 \times 10^{-4}-3 \times 10^{-9}$;

$$
2-p_{10}=1.7 \times 10^{-4}-1.7 \times 10^{-8}
$$

It follows from Fig. 4 that as the probability value $\left(p_{10}\right)$ decreases, the probability of a detected error of the Fibonacci code in a closed-form expression decreases. It also follows from Fig. 3 that when one uses the probabilities $p_{10}=3 \times 10^{-4}-3 \times 10^{-9}$, the probability of a detected error in the Fibonacci code in a closed-form expression is higher than that when using the probabilities of data transfer in the range of $p_{10}=1.7 \times 10^{-4}-1.7 \times 10^{-8}$.

Using expression (5), we derived a probability dependence of the proper data transmission $C$ of the Fibonacci code in a closed-form expression on the code bit size $n$.

Fig. 5 shows the graph of a probability dependence of the proper transmission $C$ of the Fibonacci code in a closed-form expression on the code bit size $n$ for:

1) $p_{10}=3 \times 10^{-6}, p_{01}=3 \times 10^{-5}$

2) $p_{10}=1.7 \times 10^{-7}, p_{01}=1.7 \times 10^{-6}$.

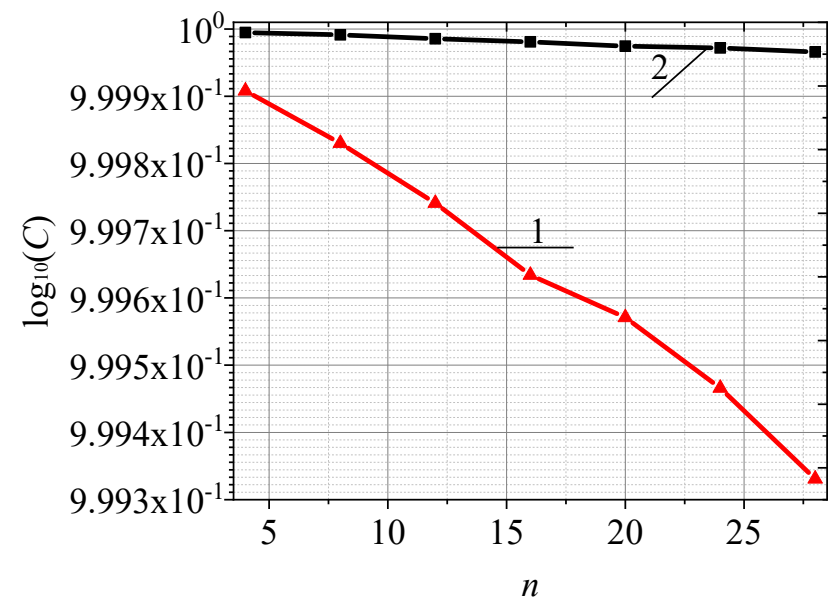

Fig. 5. Probability of the proper transfer of the Fibonacci code in a closed-form expression: $1-p_{10}=3 \times 10^{-6}, p_{01}=3 \times 10^{-5}$; $2-p_{10}=1.7 \times 10^{-7}, p_{01}=1.7 \times 10^{-6}$

It follows from Fig. 5 that as the code bit size $n$ increases, the number of bits in which an error is possible increases. Thus, the probability of proper transfer decreases with an increase in the code bit size $n$. It also follows from Fig. 5 that when using probabilities $p_{10}=1.7 \times 10^{-7}, p_{01}=1.7 \times 10^{-6}$, the probability of proper data transfer $C$ is higher than that when using probabilities $p_{10}=3 \times 10^{-6}, p_{01}=3 \times 10^{-5}$.

Using expression (6), we derived a probability dependence of an undetectable error $V$ of the Fibonacci code in a closed-form expression on the code bit size $n$.

Fig. 6 shows the graph of a probability dependence of an undetectable error of the Fibonacci code in a closed-form expression on the code bit size $n$ for:

1) $p_{10}=3 \times 10^{-6}, p_{01}=3 \times 10^{-5}$

2) $p_{10}=1.7 \times 10^{-7}, p_{01}=1.7 \times 10^{-6}$.

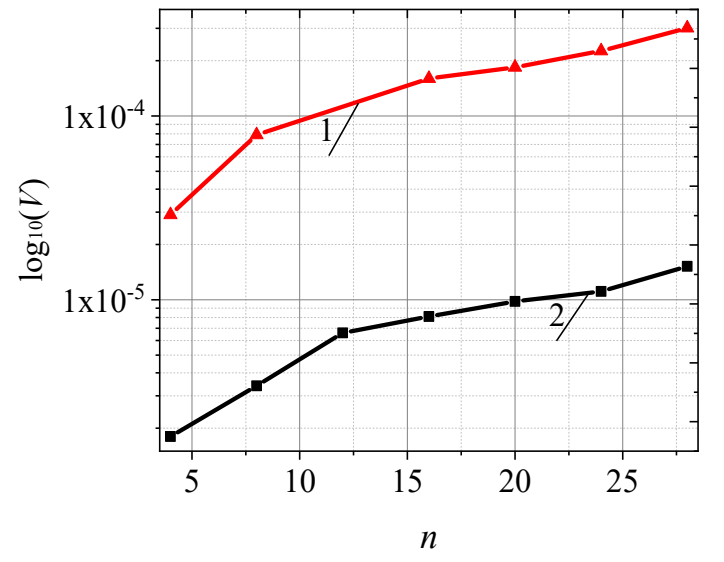

Fig. 6. Probability of an undetectable error of the Fibonacci code in a closed-form expression: $1-p_{10}=3 \times 10^{-6}, p_{01}=3 \times 10^{-5}$; $2-p_{10}=1.7 \times 10^{-7}, p_{01}=1.7 \times 10^{-6}$

It follows from Fig. 6 that the probability of an undetectable error of the Fibonacci code in a closed-form expression increases as the code bit size $n$ increases. It also follows from Fig. 6 that if one uses the probabilities $p_{10}=1.7 \times 10^{-7}$, $p_{01}=1.7 \times 10^{-6}$, the probability of an undetected error $V$ of the Fibonacci code in a closed-form expression is lower than that when using probabilities $p_{10}=3 \times 10^{-6}, p_{01}=3 \times 10^{-5}$. 
Using expression (8), we derived a probability dependence of the detected error of the Fibonacci code in a closedform expression on the code bit size $n$.

Fig. 7 shows the graph of a probability dependence of the detected error of the Fibonacci code in a closed-form expression on the code bit size $n$ for:

1) $p_{10}=3 \times 10^{-6}, p_{01}=3 \times 10^{-5}$;

2) $p_{10}=1.7 \times 10^{-7}, p_{01}=1.7 \times 10^{-6}$.

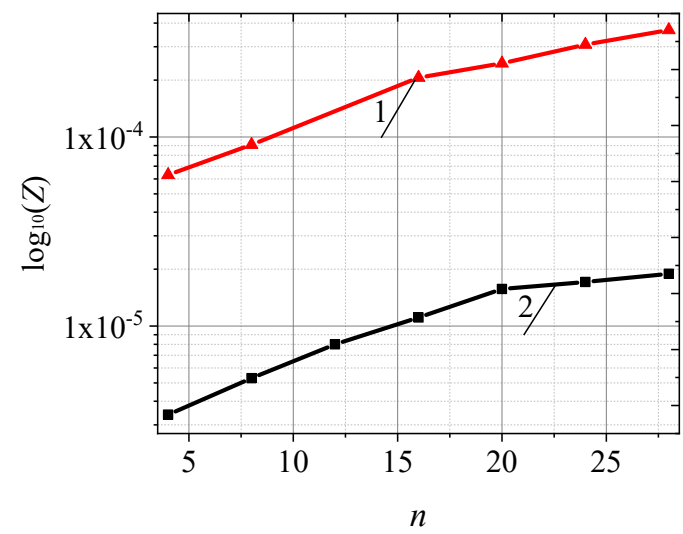

Fig. 7. Probability of a detected error of the Fibonacci code in a closed-form expression: $1-p_{10}=3 \times 10^{-6}, p_{01}=3 \times 10^{-5}$; $2-p_{10}=1.7 \times 10^{-7}, p_{01}=1.7 \times 10^{-6}$

It follows from Fig. 7 that the probability of a detected error of the Fibonacci code in a closed-form expression increases as the code bit size $n$ increases. It also follows from Fig. 7 that if one uses the probabilities $p_{10}=1.7 \times 10^{-7}$, $p_{01}=1.7 \times 10^{-6}$, a detectable error of the Fibonacci code in a closed-form expression is less than that when using probabilities $p_{10}=3 \times 10^{-6}, p_{01}=3 \times 10^{-5}$.

6. 2. Estimating the noise immunity in processing and transfer of information for the indivisible error-detecting codes

Based on an average probability method, we estimated the probability of an undetectable error for the Fibonacci code in a closed-form expression, for a parity check code, and for a constant weight code.

Fig. 8 shows the graph of a probability dependence of an undetectable error for:

1) the Fibonacci code in a closed-form expression, for $p_{10}=3 \times 10^{-4}-3 \times 10^{-9}, p_{10}=1.7 \times 10^{-4}-1.7 \times 10^{-8}$;

2) a parity check code, for $p_{10}=3 \times 10^{-4}-3 \times 10^{-9}$, $p_{10}=1.7 \times 10^{-4}-1.7 \times 10^{-8}$;

3) a constant weight code at $n=11, k=7$, for $p_{10}=3 \times 10^{-4}-3 \times 10^{-9}, p_{10}=1.7 \times 10^{-4}-1.7 \times 10^{-8}$ on $\log _{10}\left(p_{10}\right)$.

It follows from Fig. 8 that the most effective is the constant weight code, whose probability of an undetectable error is $V=1.9 \times 10^{-15}$, at $p_{10}=3 \times 10^{-9}$. Closer approximate to it is the parity check code, whose probability of an undetectable error is $V=7.7 \times 10^{-15}$, at $p_{10}=3 \times 10^{-9}$. The probability of an undetectable error of the Fibonacci code in a closed-form expression is $V=5 \times 10^{-7}$, at $p_{10}=3 \times 10^{-9}$.

The above study makes it possible, for each range of values $\left(p_{10}\right)$ and $\left(p_{01}\right)$, to calculate the probability of the proper data transmission $C$, as well as the probability of an undetectable error $V$, and a detectable error $Z$. This assessment of codes based on an average probability method allows us to assess the reliability and effectiveness of the indivisible error detecting codes, such as the Fibonacci code in a closed-form expression, a parity check code, a constant weight code, which can be effectively applied in telecommunication systems.

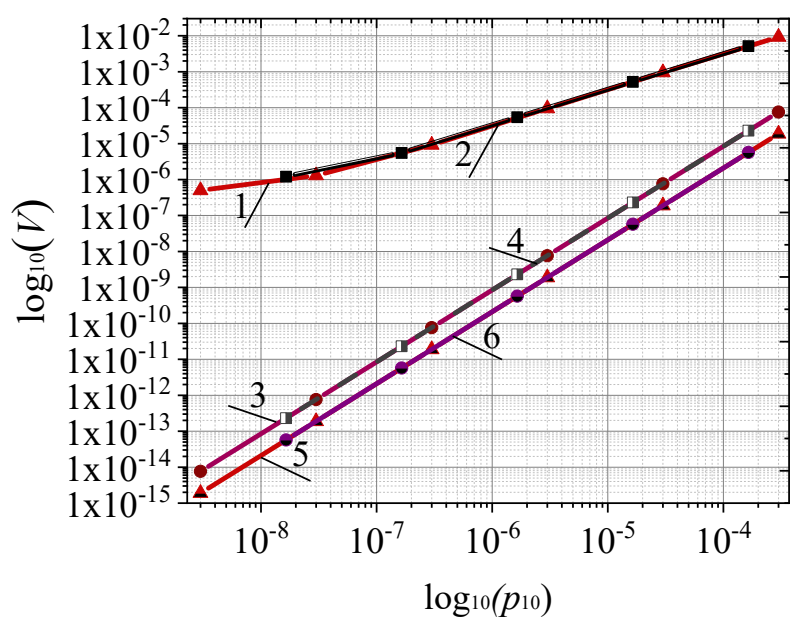

Fig. 8. Probability of an undetectable error of the Fibonacci code in a closed-form expression,

for $1-p_{10}=3 \times 10^{-4}-3 \times 10^{-9}, 2-p_{10}=1.7 \times 10^{-4}-1.7 \times 10^{-8}$; a parity check code, for $3-p_{10}=3 \times 10^{-4}-3 \times 10^{-9}$,

$4-p_{10}=1.7 \times 10^{-4}-1.7 \times 10^{-8}$; a constant weight code, for $5-p_{10}=3 \times 10^{-4}-3 \times 10^{-9}, 6-p_{10}=1.7 \times 10^{-4}-1.7 \times 10^{-8}$

7. Discussion of results of studying the indivisible errordetecting codes based on an average probability method

For the Fibonacci code in a closed-form expression, with weights $1,2,3,5,8$, for $n=5$, the number of the allowable code combinations was $M=13$, the number of the prohibited code combinations was $F=19$. The quantity of the numbers of the allowable code combinations of the Fibonacci code in a closed-form expression in the range of $n=3, \ldots, 32$ was $M=5-2.416 \times 10^{7}$. The number of the prohibited code combinations of the Fibonacci code in a closed-form expression for the range of $n=3, \ldots, 32$ was $F=3-4.289 \times 10^{9}$. We have determined the probability of transfers of the Fibonacci code combinations to the classes of proper $C$ and prohibited $Z$ ones, for the ranges of $p_{10}=3 \times 10^{-4}-3 \times 10^{-9}$ and $p_{10}=1.7 \times 10^{-4}-1.7 \times 10^{-8}$, and for the allowable ones $V$, for the ranges of $p_{01}=3 \times 10^{-3}-3 \times 10^{-8}$ and $p_{01}=1.7 \times 10^{-3}-1.7 \times 10^{-7}$.

Our assessment of the error-detecting capability of the indivisible error-detecting codes based on the average probability method makes it possible to evaluate codes that can be effectively applied in different telecommunications and digital systems in the future. It follows from our assessment that the most effective code to use is the constant weight code as this type of coding demonstrates the lowest level of an undetectable error, $V=1.9 \times 10^{-15}$, at $p_{10}=3 \times 10^{-9}$, and ensures the necessary level of reliability and error-reducing data capability.

For indivisible codes of any type offered in this paper, an assessment of the error-detecting capability of the indivisible error-detecting codes based on the average probability method has been applied. The results of applying the estimation of the indivisible error-detecting codes based on the averaged probability method have been illustrated with specific examples with the transition probabilities $0 \rightarrow 1$ and $1 \rightarrow 0$. In 
contrast to the assessment of the reliability of data transmission using the Hamming metric, this method is more general and more convenient for the indivisible error-detecting codes. The estimation of the error-detecting ability of indivisible error-detecting codes based on the averaged probability method allows estimating indivisible codes with any code distance, starting from $d=1$, which cannot be done using the Hamming metric [22]. At the same time, using the Hamming metric to assess the error-detecting ability of codes, it is possible to detect not all types of errors but only some of them, while the use of the averaged probability method makes it possible to more efficiently and accurately evaluate the indivisible error-detecting codes. In addition, the method proposed in papers $[23,24]$ to evaluate the error detecting capability of binomial coefficient-based codes is quite complex and produces worse results in terms of error detection accuracy than the average probability method, which demonstrates the simplicity, accuracy, and commonality of evaluating the indivisible codes of error detection. In the long term, the method of evaluating the indivisible codes based on average probability may be improved for divisible linear block codes.

The method of assessing indivisible codes based on average probability makes it possible to effectively evaluate and compare codes relative to each other in terms of error-detecting capability. In comparison with other methods for evaluating codes, the method for evaluating indivisible codes based on the averaged probability makes it possible to obtain the averaged probability of error on the set of initial probabilities of messages and the probabilities of transitions $0 \rightarrow 1$ and $1 \rightarrow 0$ when transmitting messages. The advantage of the averaged probability method is the simplicity of evaluating the error-detecting ability of codes and good reliability of the estimate, close to reality. In addition, a given method is universal for indivisible codes.

The disadvantage of the method of evaluating indivisible codes based on average probability is the difficulty of its application and constraints for linear block codes. In addition, the disadvantage of a given method compared to others is its increased complexity and the need to first know the probabilities of transitions $0 \rightarrow 1$ and $1 \rightarrow 0$, and, for greater accuracy, the probability of code combinations at the input to communication channels.

In the future, the method of evaluating indivisible codes based on average probability can be modified and effectively applied to divisible codes. It is also possible to use this assessment method for multi-digit codes.

\section{Conclusions}

1. Indivisible error-detecting codes have been evaluated based on the average probability method for the Fibonacci code in a closed-form expression. Detection of errors based on the average probability method makes it possible to evaluate indivisible codes with any code distance, starting from the distance of $d=1$.

2. We have performed a comparative assessment of the probability of an undetectable error for the Fibonacci code in a closed-form expression, a parity check code, and a permanent-weight code, by using an average probability method. Applying the average probability method makes it possible to estimate the probability of errors, both in processing and in the transmission of indivisible codes, as well as compare them based on this indicator.

\section{Acknowledgments}

This work has been supported by the European Regional Development Fund within the Activity 1.1.1.2 "Post-doctoral Research Aid" of the Specific Aid Objective 1.1.1 "To increase the research and innovative capacity of scientific institutions of Latvia and the ability to attract external financing, investing in human resources and infrastructure" of the Operational Program "Growth and Employment” (No. 1.1.1.2/VIAA/3/19/421).

\section{References}

1. Lin, S., Costello, D. J. (1983). Error control coding: fundamentals and applications. Prentice-Hall, 603.

2. Morelos-Zaragoza, R. H. (2006). The art of error correcting coding. John Wiley \& Sons, Inc., 278. doi: https://doi.org/ 10.1002/0470035706

3. MacWilliams, F., Sloane, N. (Eds.) (1977). The theory of Error-Correcting Codes. North Holland, 762. doi: https://doi.org/10.1016/ s0924-6509(08)x7030-8

4. Tomlinson, M., Tjhai, C. J., Ambroze, M. A., Ahmed, M., Jibril, M. (2017). Error-Correction Coding and Decoding. Springer. doi: https://doi.org/10.1007/978-3-319-51103-0

5. Arikan, E. (2009). Channel Polarization: A Method for Constructing Capacity-Achieving Codes for Symmetric Binary-Input Memoryless Channels. IEEE Transactions on Information Theory, 55 (7), 3051-3073. doi: https://doi.org/10.1109/tit.2009.2021379

6. Schlegel, C. B., Perez, L. C. (Eds.) (2015). Trellis and Turbo Coding: Iterative and Graph-Based Error Control Coding. John Wiley \& Sons, Inc, 528. doi: https://doi.org/10.1002/9781119106319

7. Borysenko, A. A., Horiachev, O. Y., Matsenko, S. M., Kobiakov, O. M. (2018). Noise-immune codes based on permutations. 2018 IEEE 9th International Conference on Dependable Systems, Services and Technologies (DESSERT). doi: https://doi.org/10.1109/ dessert.2018.8409204

8. Faure, E. V. (2017). Factorial coding with error correction. Radio Electronics, Computer Science, Control, 3, 130-138. doi: https:// doi.org/10.15588/1607-3274-2017-3-15

9. Borysenko, O., Kalashnikov, V., Kalashnykova, N. (2016). Description and applications of binomial numeral systems. Computer Science and Cyber Security, 2 (2), 13-21.

10. Grinenko, V. V. (2004). Otsenka pomehoustoychivosti binomial'nyh modifitsirovannyh kodov. Visnyk Sumskoho derzhavnoho universytetu. Seriya: Tekhnichni nauky, 12 (71), 64-69. 
11. Borysenko, O., Matsenko, S., Spolitis, S., Bobrovs, V. (2020). Development of the Fibonacci-Octal Error Detection Code for Telecommunication Systems. 2020 24th International Conference Electronics. doi: https://doi.org/10.1109/ ieeeconf 49502.2020 .9141620

12. Zeger, K., Vardy, A., Agrell, E. (2000). Upper bounds for constant-weight codes. IEEE Transactions on Information Theory, 46 (7), 2373-2395. doi: https://doi.org/10.1109/18.887851

13. Wang, X.-M., Yang, Y.-X. (1994). On the undetected error probability of nonlinear binary constant weight codes. IEEE Transactions on Communications, 42 (7), 2390-2394. doi: https://doi.org/10.1109/26.297847

14. Hoggatt, V. (1969). Fibonacci and Lucas Numbers. MA: Houghton Mifflin, 92.

15. Vajda, S. (1989). Fibonacci \& Lucas Numbers, and the Golden Section: Theory and Applications. Chichester: Ellis Horwood Ltd, 189.

16. Vorobyov, N. (1966). The Fibonacci Numbers. DC Heath, 47.

17. Stakhov, A. (2016). Fibonacci p-codes and Codes of the "Golden" p-proportions: New Informational and Arithmetical Foundations of Computer Science and Digital Metrology for Mission-Critical Applications. British Journal of Mathematics \& Computer Science, 17 (1), 1-49. doi: https://doi.org/10.9734/bjmcs/2016/25969

18. Avila, B. T., Campello de Souza, R. M. (2017). Meta-Fibonacci Codes: Efficient Universal Coding of Natural Numbers. IEEE Transactions on Information Theory, 63 (4), 2357-2375. doi: https://doi.org/10.1109/tit.2017.2663433

19. Borysenko, O., Kulyk, I., Matsenko, S., Berezhna, O., Matsenko, A. (2016). Optimal synthesis of digital counters in the Fibonacci codes with the minimal form of representation. Eastern-European Journal of Enterprise Technologies, 4 (4 (82)), 4-10. doi: https:// doi.org/10.15587/1729-4061.2016.75596

20. Matsenko, S., Borysenko, O., Spolitis, S., Bobrovs, V. (2019). Noise Immunity of the Fibonacci Counter with the Fractal Decoder Device for Telecommunication Systems. Latvian Journal of Physics and Technical Sciences, 56 (5), 12-21. doi: https://doi.org/ 10.2478/lpts-2019-0027

21. Borisenko, A. A., Onanchenko, E. L. (1994). Otsenka pomehoustoychivosti nerazdelimyh kodov. Visnyk Sumskoho universytetu, $2,64-68$.

22. Hamming, R. W. (1950). Error Detecting and Error Correcting Codes. Bell System Technical Journal, 29 (2), 147-160. doi: https:// doi.org/10.1002/j.1538-7305.1950.tb00463.x

23. Gorbunov, E. (1956). Sravnenie nekotoryh pomehoustoychivyh kodov. Telekommunikatsii, 12, 42-47.

24. Gordeev, N. (1957). O vybore dliny koda dlya simpleksnogo kanala peredachi dannyh. Teoreticheskiy uchebnik uchrezhdeniy svyazi, $72,19-25$. 